\title{
The effect of sodium glucose cotransporter 2 inhibition with empagliflozin on microalbuminuria and macroalbuminuria in patients with type 2 diabetes
}

\author{
David Cherney $^{1,2}$ • Søren S. Lund ${ }^{3}$ • Bruce A. Perkins ${ }^{4}$ - Per-Henrik Groop ${ }^{5,6,7}$. \\ Mark E. Cooper ${ }^{7} \cdot$ Stefan Kaspers $^{3} \cdot$ Egon Pfarr $^{3} \cdot$ Hans J. Woerle ${ }^{3}$. \\ Maximilian von Eynatten ${ }^{3}$
}

Received: 11 January 2016/Accepted: 13 May 2016/Published online: 17 June 2016

(C) Springer-Verlag Berlin Heidelberg 2016

\begin{abstract}
Aims/hypothesis Sodium glucose cotransporter 2 (SGLT2) inhibition lowers $\mathrm{HbA}_{1 \mathrm{c}}$, systolic $\mathrm{BP}$ (SBP) and weight in patients with type 2 diabetes and reduces renal hyperfiltration associated with type 1 diabetes, suggesting decreased intraglomerular hypertension. As lowering $\mathrm{HbA}_{1 \mathrm{c}}$, SBP, weight and intraglomerular pressure is associated with antialbuminuric effects in diabetes, we hypothesised that SGLT2 inhibition would reduce the urine albumin-to-creatinine ratio (UACR) to a clinically meaningful extent.

Methods We examined the effect of the SGLT2 inhibitor empagliflozin on UACR by pooling data from patients with type 2 diabetes and prevalent microalbuminuria $(\mathrm{UACR}=$
\end{abstract}

David Cherney and Søren S. Lund contributed equally to this article.

Electronic supplementary material The online version of this article (doi:10.1007/s00125-016-4008-2) contains peer-reviewed but unedited supplementary material, which is available to authorised users.

David Cherney

david.cherney@uhn.ca

1 Toronto General Hospital, 585 University Ave, 8N-845, Toronto, ON, Canada M5G 2N2

2 Department of Medicine, Division of Nephrology, and Department of Physiology, University of Toronto, Toronto, ON, Canada

3 Boehringer Ingelheim Pharma GmbH \& Co. KG, Ingelheim, Germany

4 Mount Sinai Hospital, University of Toronto, Toronto, ON, Canada

5 Abdominal Centre Nephrology, University of Helsinki and Helsinki University Hospital, Helsinki, Finland

6 Folkhälsan Research Centre, Biomedicum Helsinki, Helsinki, Finland

7 Baker IDI Heart and Diabetes Institute, Melbourne, VIC, Australia
$30-300 \mathrm{mg} / \mathrm{g} ; n=636)$ or macroalbuminuria (UACR $>300 \mathrm{mg} / \mathrm{g} ; n=215$ ) who participated in one of five phase III randomised clinical trials. Primary assessment was defined as percentage change in geometric mean UACR from baseline to week 24 .

Results After controlling for clinical confounders including baseline log-transformed UACR, $\mathrm{HbA}_{1 \mathrm{c}}$, SBP and estimated GFR (according to the Modification of Diet in Renal Disease [MDRD] formula), treatment with empagliflozin significantly reduced UACR in patients with microalbuminuria $(-32 \%$ vs placebo; $p<0.001)$ or macroalbuminuria $(-41 \%$ vs placebo; $p<0.001)$. Intriguingly, in regression models, most of the UACR-lowering effect with empagliflozin was not explained by SGLT2 inhibition-related improvements in $\mathrm{HbA}_{1 \mathrm{c}}$, $\mathrm{SBP}$ or weight.

Conclusions/interpretation In patients with type 2 diabetes and either micro- or macroalbuminuria, empagliflozin reduced UACR by a clinically meaningful amount. This effect was largely independent of the known metabolic or systemic haemodynamic effects of this drug class. Our results further support a direct renal effect of SGLT2 inhibitors. Prospective studies are needed to explore the potential of this intervention to alter the course of kidney disease in high-risk patients with diabetes.

Trial registration: Clinicaltrials.gov NCT01177813 (study 1); NCT01159600 (study 2); NCT01159600 (study 3); NCT01210001 (study 4); and NCT01164501 (study 5).

Keywords Empagliflozin · Macroalbuminuria . Microalbuminuria $\cdot$ Sodium glucose cotransporter 2 . Type 2 diabetes $\cdot$ Urine albumin-to-creatinine ratio
Abbreviations
AE Adverse event
CKD Chronic kidney disease 


$\begin{array}{ll}\text { DBP } & \text { Diastolic BP } \\ \text { eGFR } & \text { Estimated GFR } \\ \text { ESRD } & \text { End-stage renal disease } \\ \text { FAS } & \text { Full analysis set } \\ \text { LOCF } & \text { Last observation carried forward } \\ \text { MDRD } & \text { Modification of Diet in Renal Disease } \\ \text { PP } & \text { Pulse pressure } \\ \text { RAAS } & \text { Renin-angiotensin-aldosterone system } \\ \text { SBP } & \text { Systolic BP } \\ \text { SGLT2 } & \text { Sodium glucose cotransporter 2 } \\ \text { TS } & \text { Treated set } \\ \text { UACR } & \text { Urine albumin-to-creatinine ratio }\end{array}$

\section{Introduction}

Diabetes is the leading cause of end-stage renal disease (ESRD) in the USA, and accounts for $>40 \%$ of patients requiring renal replacement therapy [1]. Current clinical indicators of underlying diabetic kidney disease and progression of nephropathy are the presence and worsening degree of albuminuria and/or progressive renal function decline. Although not an ideal biomarker of renal risk, the presence of microalbuminuria has been linked to an increased risk for both cardiovascular events and progression of kidney disease in patients with type 2 diabetes. Macroalbuminuria is more consistently associated with an elevated risk of both renal and cardiovascular complications [2, 3]. Therefore, regular assessment of albuminuria remains a clinical cornerstone to diagnose the onset of kidney disease in diabetes and to prospectively track the progression of renal injury.

The current standard renal protective therapies in diabetes involve glycaemic and BP control, and blockade of the reninangiotensin-aldosterone system (RAAS) [4]. Therapeutic options for patients with persistent albuminuria in the setting of satisfactory $\mathrm{HbA}_{1 \mathrm{c}}$ and $\mathrm{BP}$ control are limited to changes in lifestyle, such as sodium restriction and weight loss, which are supported by limited evidence and are often difficult to achieve $[5,6]$.

Empagliflozin is a highly selective sodium glucose cotransporter 2 (SGLT2) inhibitor. In patients with type 2 diabetes, empagliflozin is consistently associated with declines in $\mathrm{HbA}_{1 \mathrm{c}}$, systolic BP (SBP) and weight [7-10]. Empagliflozin has been shown to decrease renal hyperfiltration in patients with type 1 diabetes [11]. This renal physiological characteristic has been linked to the development of nephropathy in type 1 and type 2 diabetes [12-14]. Furthermore, SGLT2 inhibition may improve this early glomerular haemodynamic abnormality through inhibition of sodium-glucose reabsorption at the proximal tubule [12-14]. Such a natriuretic effect increases sodium delivery to the distal tubule, thereby stimulating tubuloglomerular feedback, ultimately causing afferent renal arteriolar vasoconstriction and a reduction in intraglomerular pressure $[11,12]$. Based on available data in healthy humans and in patients with type 1 diabetes, the renal haemodynamic effects of SGLT2 inhibition appear to be RAAS independent, as plasma and urinary levels of RAAS mediators increase modestly, rather than decline, in response to the intervention $[11,13,14]$. The renal protective effect of empagliflozin, including effects on established clinical renal biomarkers such as albuminuria, remains to be systematically studied.

We therefore examined the effect of 24 weeks' treatment with empagliflozin on urine albumin-to-creatinine ratio (UACR) in patients with type 2 diabetes and either microalbuminuria (UACR $=30-300 \mathrm{mg} / \mathrm{g}$ ) or macroalbuminuria (UACR $>300 \mathrm{mg} / \mathrm{g}$ ) who participated in one of five phase III randomised placebo-controlled clinical trials. We hypothesised that adding empagliflozin to a background of glucose- and BPlowering therapy would reduce UACR compared with placebo.

\section{Methods}

Study population This pooled analysis comprised patients with type 2 diabetes who participated in one of five phase III clinical trials of empagliflozin with primary endpoints related to glucose lowering. Study design and entry criteria for all five trials have been published [7-10, 15] (see electronic supplementary material [ESM] Methods). In four studies, patients with eGFR $\geq 30 \mathrm{ml} \mathrm{min}^{-1} 1.73 \mathrm{~m}^{-2}$ were randomised to receive empagliflozin $10 \mathrm{mg}$, empagliflozin $25 \mathrm{mg}$ or placebo once daily for 24 weeks as monotherapy or add-on therapy to background glucose-lowering medication. In the fifth study (EMPA-REG RENAL), which included individuals with type 2 diabetes and chronic kidney disease (CKD), patients with CKD stage 2 received empagliflozin $10 \mathrm{mg}$, empagliflozin $25 \mathrm{mg}$ or placebo, and patients with CKD stage 3 or stage 4 received empagliflozin $25 \mathrm{mg}$ or placebo once daily for 52 weeks as add-on therapy [15]. UACR at 24 weeks was used in this analysis to remove the potential confounder of treatment length across trials.

Urine samples were taken at screening, the start of placebo run-in, baseline, week 12 and week 24. Urinary albumin and creatinine concentrations at respective time points were measured from a single standardised spot urine sample and UACR was calculated at the central laboratory. UACR was therefore measured once per visit in a morning sample, in the fasted state and before intake of trial medication. In the total pooled population $(n=3215$ ), microalbuminuria (UACR $=30-300 \mathrm{mg} / \mathrm{g}$ ) was present in $636(19.8 \%)$ individuals at baseline (248 patients who received placebo, 159 patients who received empagliflozin $10 \mathrm{mg}$ and 229 patients who received empagliflozin $25 \mathrm{mg}$ ). In addition, macroalbuminuria (UACR $>300 \mathrm{mg} / \mathrm{g}$ ) was identified in $215(6.7 \%)$ individuals at baseline (87 patients receiving placebo, 36 patients receiving empagliflozin $10 \mathrm{mg}$ and 92 patients receiving empagliflozin $25 \mathrm{mg}$ ). 
Endpoints The primary assessment of this pooled analysis was defined as percentage change in geometric mean of the UACR from baseline to week 24 as described for other drug interventions targeting albuminuria [16, 17]. Empagliflozin 10 and $25 \mathrm{mg}$ doses were pooled to define the overall magnitude of the drug effect compared with placebo.

Other efficacy endpoints were defined as change in $\mathrm{HbA}_{1 \mathrm{c}}$, weight and SBP from baseline to week 24 . Selected safety endpoints were changes in estimated GFR (eGFR) (Modification of Diet in Renal Disease [MDRD]) over 24 weeks and overall adverse events (AEs), including the incidence of AEs related to volume depletion and electrolyte imbalance, such as hyperkalaemia.

Statistical methods The treated set (TS) comprised the pooled population of all randomised patients from the five studies who received at least one dose of study drug and were identified with prevalent albuminuria at baseline. All efficacy analyses were performed on the full analysis set (FAS), which included all patients from the TS who had documented baseline $\mathrm{HbA}_{1 \mathrm{c}}$ and UACR values. Any efficacy data collected following initiation of hyperglycaemic rescue therapy were set to missing. Missing data were imputed using a last observation carried forward (LOCF) approach.

For all efficacy analyses, UACR data were initially $\log _{10}$ transformed because of the left-skewed distribution. Statistical procedures were conducted separately for patients with microor macroalbuminuria at baseline. Changes in UACR from baseline to week 24 were analysed by ANCOVA and adjusted for $\log _{10}$-transformed UACR at baseline, alongside baseline values for $\mathrm{HbA}_{1 \mathrm{c}}$, eGFR (MDRD), SBP, region, treatment and trial effect. The values were then back-transformed to obtain geometric means of the UACR ratios for depiction of 24-week treatment effects (with values expressed as percentage change in adjusted geometric mean of the UACR ratios). Further multivariable models were employed to explore the potential impact of concomitant treatment changes in $\mathrm{HbA}_{1 \mathrm{c}}$, weight, SBP, diastolic $\mathrm{BP}$ (DBP) or pulse pressure (PP) and the combined effect of changes in $\mathrm{HbA}_{1}$, weight and SBP on UACR effects at week 24. We used individual ANCOVA models with baseline $\mathrm{HbA}_{1 \mathrm{c}}$, SBP, log-transformed UACR, the baseline of the variable of interest, and the change from baseline in the variable or variables of interest as linear covariates in addition to the following fixed effects: treatment, baseline eGFR (MDRD), region and trial.

Changes in $\mathrm{HbA}_{1 \mathrm{c}}$, weight and SBP at week 24 were evaluated for the FAS using ANCOVA. Here, baseline $\mathrm{HbA}_{1 \mathrm{c}}$ and the respective baseline value of the endpoint in question were used as linear covariates. For all analyses, baseline eGFR (MDRD), region, trial and treatment were included as fixed effects. Analyses for change in eGFR (MDRD) over 24 weeks and frequencies of AEs were descriptive and based on the TS.

\section{Results}

Study population and baseline characteristics Baseline characteristics are shown in Table 1. As expected, individuals with macroalbuminuria tended to have longer diabetes duration, higher SBP and lower eGFR (MDRD) compared with those with microalbuminuria. In microalbuminuric patients, between $50 \%$ and $60 \%$ of patients were taking RAAS inhibitors at baseline. Macroalbuminuric patients tended to use more anti-hypertensive agents, including RAAS inhibitors, and to take lipid-lowering and anti-platelet agents more frequently. Within the microalbuminuric and macroalbuminuric groups, patients allocated to placebo or empagliflozin were well balanced in terms of demographic factors, background anti-hypertensive therapies, metabolic variables, BP and level of albuminuria. Baseline eGFR was not significantly different between empagliflozin and placebo groups in patients with microalbuminuria or macroalbuminuria at baseline $(p=0.475$ and $p=0.450$, respectively). In the total study cohort, the proportion of patients with a medical history of diabetic retinopathy was $27.4 \%$ in the trial involving patients with $\mathrm{CKD}$, and ranged from $1.4 \%$ to $10.2 \%$ in the other four studies.

Effects of empagliflozin on $\mathrm{HbA}_{1 \mathrm{c}}$, weight and BP As expected, empagliflozin was associated with significant placebocorrected declines in $\mathrm{HbA}_{1 \mathrm{c}}$ (ESM Fig. 1a, b), weight (ESM Fig. 1c, d), SBP (ESM Fig. 1e, f) and DBP (ESM Fig. 1g, h) in groups of patients with micro- or macroalbuminuria.

\section{UACR effects of SGLT2 inhibition with empagliflozin} After 24 weeks of treatment with empagliflozin, UACR values were significantly decreased compared with placebo in the microalbuminuria group (treatment difference $-32 \%[-41 \%$ to $-22 \%$ ]; $p<0.001$, Fig. 1a) and the macroalbuminuria group $(-41 \%$ [ $-57 \%$ to $-19 \%] ; p<0.01$, Fig. $1 b)$. A pronounced effect of empagliflozin on UACR in both albuminuria groups was observed with the first 12 weeks of treatment, and the effects were maintained for the remainder of the 24-week treatment period (ESM Fig. 2). The estimated effects were almost identical in a sensitivity analysis including baseline DBP, weight, sex, BMI, race, weight and baseline RAAS inhibitor use (data not shown). Replacing DBP with PP as a surrogate marker of arterial stiffness in this enriched model also resulted in the same effect (data not shown).

To also explore potential differential effects on UACR between the two empagliflozin doses, additional sensitivity analyses of the primary endpoint were conducted for the pool of studies one to four. In patients with microalbuminuria, similar effects of empagliflozin $10 \mathrm{mg}(n=141)$ and empagliflozin $25 \mathrm{mg}(n=150)$ were observed after 24 weeks (adjusted mean difference: $-0.04,[-0.13,0.05] ; p=0.431)$. The observation of no significant dose-related difference in albuminuria was further confirmed in patients with macroalbuminuria (adjusted 
Table 1 Baseline characteristics

\begin{tabular}{|c|c|c|c|c|}
\hline \multirow[t]{2}{*}{ Characteristic } & \multicolumn{2}{|c|}{$\begin{array}{l}\text { Patients with microalbuminuria at } \\
\text { baseline }\end{array}$} & \multicolumn{2}{|c|}{$\begin{array}{l}\text { Patients with macroalbuminuria at } \\
\text { baseline }\end{array}$} \\
\hline & $\begin{array}{l}\text { Placebo } \\
(n=248)\end{array}$ & $\begin{array}{l}\text { Empagliflozin } \\
(n=388)\end{array}$ & $\begin{array}{l}\text { Placebo } \\
(n=87)\end{array}$ & $\begin{array}{l}\text { Empagliflozin } \\
(n=128)\end{array}$ \\
\hline Male & $141(56.9)$ & $229(59.0)$ & $58(66.7)$ & $86(67.2)$ \\
\hline $\mathrm{HbA}_{1 \mathrm{c}}, \%$ & $8.15(0.85)$ & $8.17(0.87)$ & $8.13(0.86)$ & $8.20(0.92)$ \\
\hline $\mathrm{HbA}_{1 \mathrm{c}}, \mathrm{mmol} / \mathrm{mol}$ & $66(9)$ & $66(10)$ & $65(9)$ & $66(10)$ \\
\hline Weight, $\mathrm{kg}$ & $79.1(19.2)$ & $80.3(19.5)$ & $80.3(20.2)$ & $82.6(18.9)$ \\
\hline $\mathrm{BMI}, \mathrm{kg} / \mathrm{m}^{2}$ & $29.2(5.6)$ & $29.4(5.6)$ & $29.8(5.6)$ & $29.7(5.1)$ \\
\hline $\mathrm{SBP}, \mathrm{mmHg}$ & $134.2(17.0)$ & $134.9(16.9)$ & $145.1(19.2)$ & $143.4(18.9)$ \\
\hline DBP, $\mathrm{mmHg}$ & $77.3(9.6)$ & $79.4(9.5)$ & $78.0(11.2)$ & $79.0(10.0)$ \\
\hline eGFR (MDRD), $\mathrm{ml} \mathrm{min} \min ^{-1} 1.73 \mathrm{~m}^{-2}$ & $72.1(30.4)$ & $79.4(26.0)$ & $52.5(29.0)$ & $56.6(29.0)$ \\
\hline \multicolumn{5}{|l|}{ eGFR (MDRD), $n(\%)$} \\
\hline$\geq 90 \mathrm{ml} \mathrm{min}-1.73 \mathrm{~m}^{-2}$ & $69(27.8)$ & $129(33.2)$ & $12(13.8)$ & $17(13.3)$ \\
\hline$\geq 60$ to $<90 \mathrm{ml} \mathrm{min}^{-1} 1.73 \mathrm{~m}^{-2}$ & $83(33.5)$ & $180(46.4)$ & $15(17.2)$ & $31(24.2)$ \\
\hline$\geq 30$ to $<60 \mathrm{ml} \mathrm{min}^{-1} 1.73 \mathrm{~m}^{-2}$ & $83(33.5)$ & $71(18.3)$ & $39(44.8)$ & $56(43.8)$ \\
\hline$<30 \mathrm{ml} \mathrm{min}^{-1} 1.73 \mathrm{~m}^{-2}$ & $13(5.2)$ & $8(2.1)$ & $21(24.1)$ & $24(18.8)$ \\
\hline $\mathrm{UACR}^{\mathrm{a}}, \mathrm{mg} / \mathrm{g}$ & $67(41-117)$ & $66(42-118)$ & $961(514-2045)$ & $874(507-1744)$ \\
\hline $\mathrm{UACR}^{\mathrm{a}}, \mathrm{mg} / \mathrm{mmol}$ & $7.6(4.6-13.3)$ & $7.5(4.7-13.4)$ & $108.7(58.2-231.3)$ & $98.9(57.4-197.3)$ \\
\hline \multicolumn{5}{|l|}{ Race } \\
\hline Asian & $132(53.2)$ & $223(57.5)$ & $49(56.3)$ & $63(49.2)$ \\
\hline White & $112(45.2)$ & $153(39.4)$ & $38(43.7)$ & $60(46.9)$ \\
\hline Other & $4(1.6)$ & $12(3.1)$ & 0 & $5(3.9)$ \\
\hline Age, years & $60.4(10.2)$ & $58.4(10.5)$ & $60.5(10.3)$ & $59.3(9.9)$ \\
\hline \multicolumn{5}{|l|}{ Time since diagnosis of $\mathrm{T} 2 \mathrm{D}$} \\
\hline$\leq 1$ year & $20(8.1)$ & $37(9.5)$ & $1(1.1)$ & $5(3.9)$ \\
\hline$>1$ to 5 years & $55(22.2)$ & $108(27.8)$ & $15(17.2)$ & $20(15.6)$ \\
\hline$>5$ years & $173(69.8)$ & $243(62.6)$ & $71(81.6)$ & $103(80.5)$ \\
\hline Background anti-hypertensive medication $^{\mathrm{b}}$ & $168(67.7)$ & $259(66.8)$ & $73(83.9)$ & $113(88.3)$ \\
\hline Agents acting on renin-angiotensin system & $142(57.3)$ & $203(52.3)$ & $57(65.5)$ & $96(75.0)$ \\
\hline ACE inhibitor, plain & $59(23.8)$ & $83(21.4)$ & $29(33.3)$ & $47(36.7)$ \\
\hline ACE inhibitor, combination $^{c}$ & $6(2.4)$ & $18(4.6)$ & $2(2.3)$ & $6(4.7)$ \\
\hline Angiotensin II antagonist, plain & $60(24.2)$ & $75(19.3)$ & $26(29.9)$ & $41(32.0)$ \\
\hline Angiotensin II antagonist, combination ${ }^{\mathrm{c}}$ & $20(8.1)$ & $32(8.2)$ & $6(6.9)$ & $10(7.8)$ \\
\hline Other & $3(1.2)$ & $2(0.5)$ & $4(4.6)$ & $5(3.9)$ \\
\hline Diuretics & $45(18.1)$ & $55(14.2)$ & $30(34.5)$ & $39(30.5)$ \\
\hline Loop diuretics & $22(8.9)$ & $16(4.1)$ & $22(25.3)$ & $25(19.5)$ \\
\hline Thiazides & $16(6.5)$ & $22(5.7)$ & $5(5.7)$ & $13(10.2)$ \\
\hline Low-ceiling diuretics, excl. thiazides & $9(3.6)$ & $13(3.4)$ & $2(2.3)$ & $6(4.7)$ \\
\hline Potassium-sparing agents & $2(0.8)$ & $4(1.0)$ & $1(1.1)$ & $2(1.6)$ \\
\hline Diuretics and potassium-sparing agents in combination & $1(0.4)$ & $2(0.5)$ & $1(1.1)$ & $1(0.8)$ \\
\hline Other diuretics & 0 & $2(0.5)$ & 0 & 0 \\
\hline Calcium channel blockers & $74(29.8)$ & $110(28.4)$ & $41(47.1)$ & $60(46.9)$ \\
\hline Beta-blockers & $49(19.8)$ & $73(18.8)$ & $32(36.8)$ & $50(39.1)$ \\
\hline Other & $14(5.6)$ & $18(4.6)$ & $18(20.7)$ & $13(10.2)$ \\
\hline Background lipid-lowering medication & $121(48.8)$ & $169(43.6)$ & $56(64.4)$ & $79(61.7)$ \\
\hline Background aspirin & $91(36.7)$ & $105(27.1)$ & $40(46.0)$ & $62(48.4)$ \\
\hline
\end{tabular}

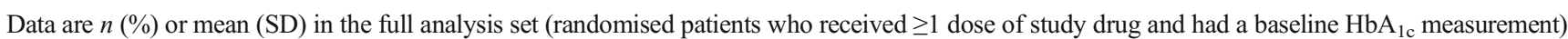
unless otherwise stated

${ }^{a}$ Median (1st quartile-3rd quartile)

${ }^{\mathrm{b}}$ Patients could be receiving $\geq 1$ type of anti-hypertensive medication

${ }^{\mathrm{c}}$ Combination with diuretics or calcium channel blockers or other combinations

T2D, type 2 diabetes

mean difference in UACR between empagliflozin $10 \mathrm{mg}$ $[n=27]$ and $25 \mathrm{mg}$ [ $n=19]$ : adjusted mean difference: -0.36 $[-0.78,0.05] ; p=0.084)$.

Contribution of changes in $\mathrm{HbA}_{1 \mathrm{c}}$, weight and $\mathrm{BP}$ on UACR effects with empagliflozin To further decipher the potential mechanisms underlying the observed decline in micro- and macroalbuminuria with empagliflozin at week 24 , we employed a comprehensive ANCOVA model. This model accounted for changes in $\mathrm{HbA}_{1 \mathrm{c}}$, weight, SBP or DBP from baseline to week 24 and thus adjusted for those variables known to be altered by SGLT2 inhibition. This is important because the 


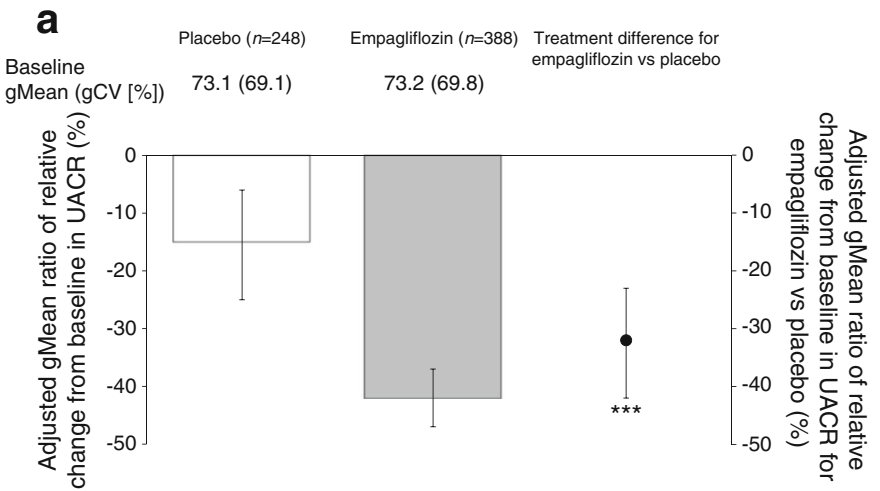

Fig. 1 Adjusted geometric mean (with 95\% CI) of percentage change from baseline in UACR at week 24 in patients with microalbuminuria (a) or macroalbuminuria (b) at baseline. ANCOVA in FAS (LOCF).

selected variables are generally also known to be associated with albuminuria in patients with type 2 diabetes [1]. This model showed that overall improvements in microalbuminuria with empagliflozin $(-32 \%)$ were not significantly influenced by concomitant changes in $\mathrm{HbA}_{1 \mathrm{c}}(-2 \%)$ (Fig. 2a and ESM Fig. 3a). In contrast, in separate models, the influence of weight $(-6 \%)$, as well as SBP (-9\%) and DBP (-7\%) made statistically significant, albeit modest, contributions to the overall UACR decline with empagliflozin (Fig. 2b-d and ESM Fig. 3b-d). In a model that included changes in $\mathrm{HbA}_{1 \mathrm{c}}$, weight and SBP simultaneously, these factors contributed a total of $15 \%$ to the overall UACR decline of $33 \%$ with empagliflozin vs placebo (Fig. 2e and ESM Fig. 3e). That is, their individual effect on UACR appeared to be somewhat additive and, in total, accounted for about half of the UACR-lowering effect of empagliflozin. Finally, in patients with microalbuminuria, changes in UACR were only weakly correlated, albeit statistically significant in some cases, with changes in $\mathrm{HbA}_{1 \mathrm{c}}$ (placebo $r=0.129, p=0.042$; empagliflozin $r=0.021, p=0.673$ ) or weight (placebo $r=0.109, p=0.088$; empagliflozin $r=0.103, p=0.043$ ). The association between UACR change and PP change was also weak, albeit statistically significant, and was similar in the two groups (placebo $r=0.143$, $p=0.024$; empagliflozin $r=0.141, p=0.006$ ). These relationships indicate that the effect on UACR was not driven by change in $\mathrm{HbA}_{1 \mathrm{c}}$, $\mathrm{PP}$ or weight, thus supporting the findings in the contribution analysis.

In patients with macroalbuminuria, changes in UACR with empagliflozin $(-41 \%)$ were not significantly influenced by change in either $\mathrm{HbA}_{1 \mathrm{c}}(+5 \%)$ or weight $(-2 \%)$ (Fig. 3a, b and ESM Fig. 4a, b). As in patients with microalbuminuria, UACR lowering with empagliflozin was significantly, but again modestly, related to changes in SBP (-9\%) and DBP (-7\%) (Fig. 3c, $\mathrm{d}$ and ESM Fig. 4c, d). In the model including changes in $\mathrm{HbA}_{1 \mathrm{c}}$, weight and SBP simultaneously, these factors contributed a total of $7 \%$ to the overall UACR decline of $40 \%$ with empagliflozin vs placebo (Fig. 3e and ESM Fig. 4e). Thus, in patients with microalbuminuria or macroalbuminuria, concomitant changes in

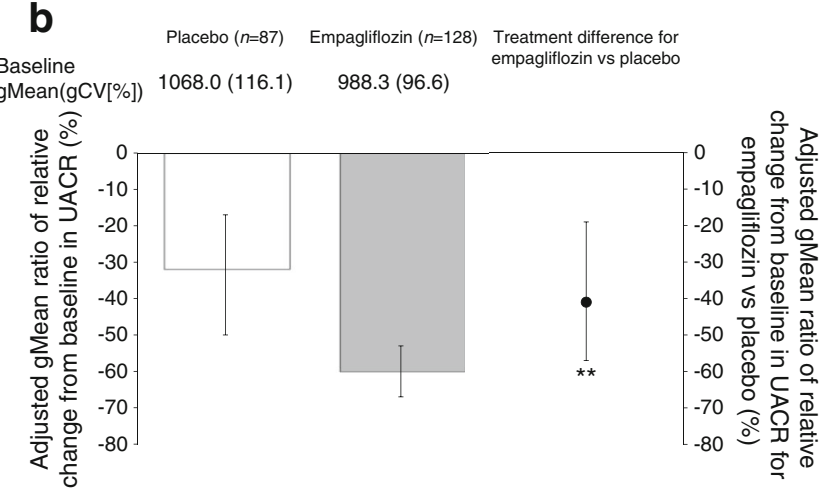

$* * p<0.01$ and $* * * p<0.001$ for treatment difference between empagliflozin and placebo. gCV, geometric $\mathrm{CV}$; gMean, geometric mean

glucose control, weight or BP individually or together only accounted for approximately half (at most) of the effect of empagliflozin on reducing albuminuria vs placebo (approximately $5-15$ percentage points out of a total reduction of approximately $30-40$ percentage points). In macroalbuminuric patients, changes in UACR were not correlated with changes in $\mathrm{HbA}_{1 \mathrm{c}}$ (placebo $r=0.133, p=0.218$; empagliflozin $r=-0.046$, $p=0.607$ ), weight (placebo $r=-0.009, p=0.934$; empagliflozin $r=0.081, p=0.366$ ) or PP (placebo $r=0.190, p=0.078$; empagliflozin $r=0.061, p=0.492)$. These relationships indicate that the effect on UACR was not driven by changes in $\mathrm{HbA}_{1}$, PP or weight, thus supporting the findings in the contribution analysis.

Sensitivity analyses for RAAS inhibitor use, completers vs non-completers and for patients with and without changes in anti-hypertensive agent are shown in the ESM (see ESM Results).

Safety and adverse events At weeks 12 and 24, eGFR showed a mild numerical decrease with empagliflozin and the reductions were similar in the micro- and macroalbuminuria groups (ESM Fig. 5a, b).

Reported AEs are shown in Table 2. The percentage of patients with urinary tract infections and genital infections was higher with empagliflozin than placebo. The percentage of patients with events consistent with volume depletion (including hypotension) was numerically higher with empagliflozin than placebo in patients with microalbuminuria $(1.0 \%$ vs $0.0 \%$ ) or macroalbuminuria $(2.3 \%$ vs $1.1 \%)$ (Table 2$)$. Of note, in this susceptible population of patients with underlying diabetic kidney disease, the percentage of patients with hyperkalaemia was similar between the empagliflozin and placebo groups (Table 2). The data suggest comparable rates of AEs in empagliflozin-treated patients taking the combination of a RAAS inhibitor and loop diuretic, with the caveat that the numbers are too small to allow reliable conclusions to be drawn (data not shown). 

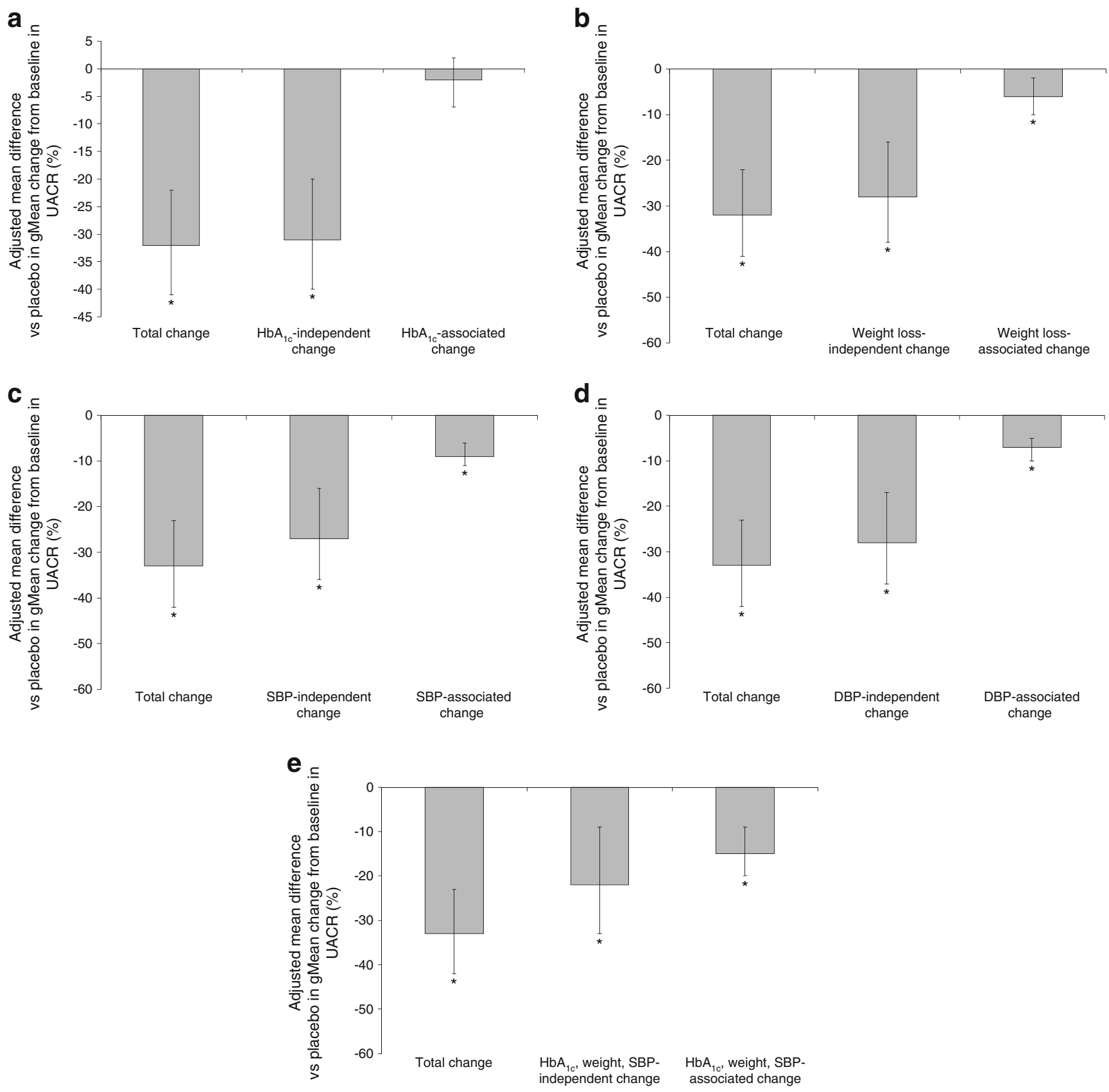

Fig. 2 Contribution of changes in $\mathrm{HbA}_{1 \mathrm{c}}$ (a), weight (b), $\mathrm{SBP}$ (c), DBP (d) and $\mathrm{HbA}_{1 \mathrm{c}}$, weight and SBP (e) to changes in UACR with empagliflozin in patients with microalbuminuria at baseline (with $95 \%$

\section{Discussion}

The main finding from this large analysis of a global clinical trial programme supports our hypothesis that SGLT2 inhibition with empagliflozin reduces UACR in both the microalbuminuric and macroalbuminuric ranges in patients with type 2 diabetes. The quantity of the observed albuminuria-lowering effect was clinically meaningful and was only partially explained by expected empagliflozin-related improvements in variables otherwise associated with albuminuria reduction, namely $\mathrm{HbA}_{1 \mathrm{c}}$, weight and
CI). Treated set (LOCF). ${ }^{*} p<0.05$ for treatment difference between empagliflozin and placebo. gMean, geometric mean

BP. In this cohort, empagliflozin was generally well tolerated, aside from increased frequencies of urinary tract and genital infections and a numerical increase in cases of volume depletion vs placebo in the micro- and macroalbuminuria groups.

Previous experimental work using different animal models of kidney disease have demonstrated that SGLT2 inhibition alleviates renal damage. In Akita and streptozotocin-induced animal models of insulin-deficient diabetes, SGLT2 inhibition reduced albuminuria [18-20]. SGLT2 inhibition has similar 

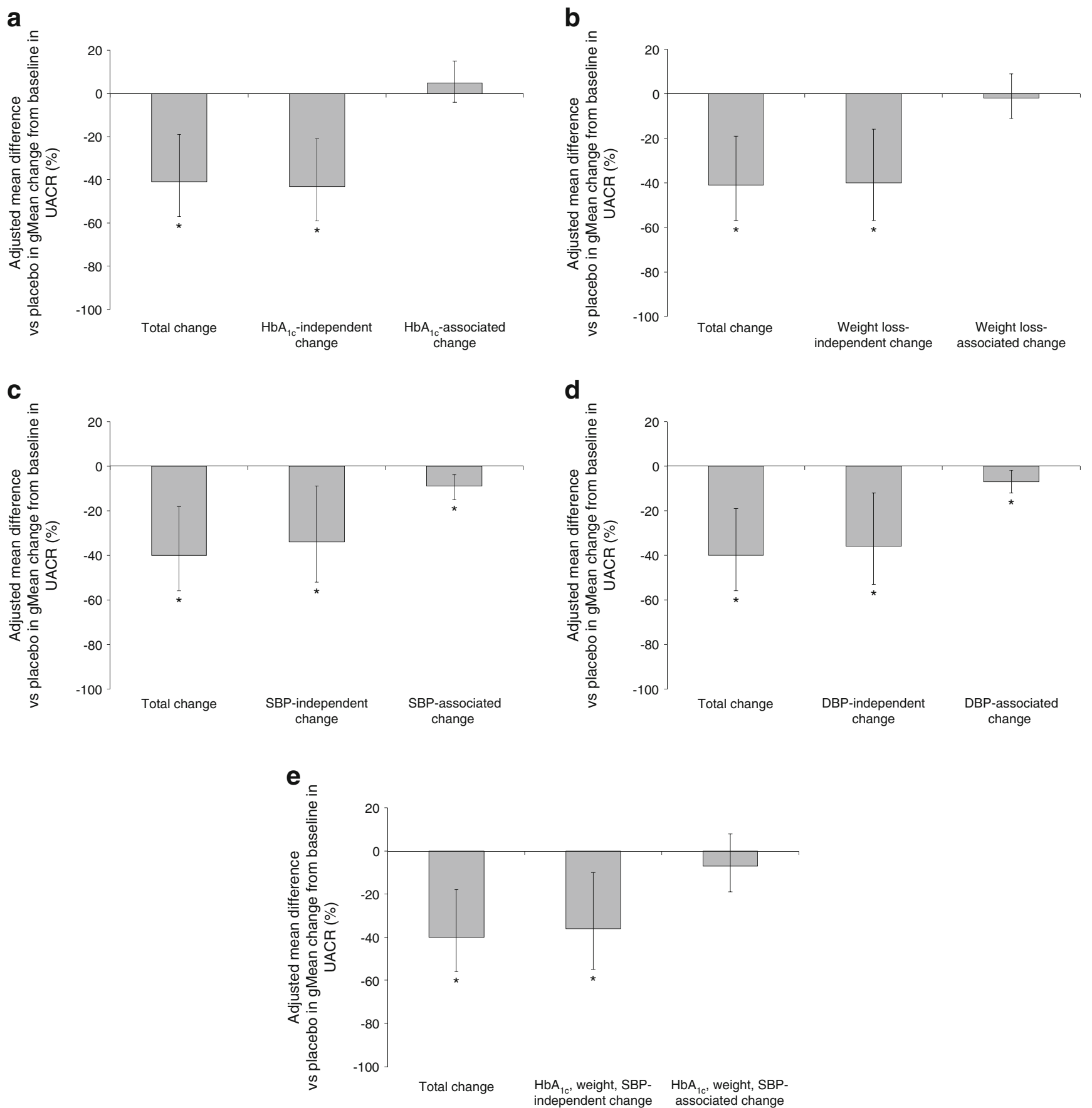

Fig. 3 Contribution of changes in $\mathrm{HbA}_{1 \mathrm{c}}$ (a), weight (b), $\mathrm{SBP}$ (c), DBP (d) and $\mathrm{HbA}_{1 \mathrm{c}}$, weight and $\mathrm{SBP}$ (e) to changes in UACR with empagliflozin in patients with macroalbuminuria at baseline (with $95 \%$

anti-albuminuric effects in animal models of type 2 diabetes [21], including recent evidence that empagliflozin reduced albuminuria, independent of effects on BP or hyperglycaemia, in BTBR $o b / o b$ mouse models of type 2 diabetes [22]. Previous clinical studies in type 2 diabetes patients with and without CKD have shown that SGLT2 inhibition is associated with an acute but modest decline in eGFR within 3-6 weeks of treatment initiation, followed by a period of stable renal function for
CI). Treated set (LOCF). ${ }^{*} p<0.05$ for treatment difference between empagliflozin and placebo. gMean, geometric mean

52-104 weeks; this change is reversible after drug cessation for 2 weeks [17, 23, 24]. Notably, within this same treatment period, renal safety assessments of SGLT2 inhibitors have also reported a reduction in UACR or urinary albumin excretion in patients with type 2 diabetes and CKD [15, 24]. Moreover, in a dedicated study of patients with stage 3 CKD, the SGLT2 inhibitor dapagliflozin failed to show a significant effect on $\mathrm{HbA}_{1 \mathrm{c}}$ at 24 weeks, yet still reduced albuminuria, BP and 
Table 2 AEs in patients with microalbuminuria or macroalbuminuria at baseline

\begin{tabular}{|c|c|c|c|c|}
\hline \multirow[t]{2}{*}{$\mathrm{AE}$} & \multicolumn{2}{|c|}{ Patients with microalbuminuria } & \multicolumn{2}{|c|}{ Patients with macroalbuminuria } \\
\hline & Placebo $(n=248)$ & Empagliflozin $(n=388)$ & Placebo $(n=87)$ & Empagliflozin $(n=128)$ \\
\hline One or more $\mathrm{AE}(\mathrm{s})$ & $180(72.6)$ & $259(66.8)$ & $68(78.2)$ & $91(71.1)$ \\
\hline One or more drug-related $\mathrm{AE}(\mathrm{s})^{\mathrm{a}}$ & $48(19.4)$ & $74(19.1)$ & $24(27.6)$ & $26(20.3)$ \\
\hline One or more $\mathrm{AE}(\mathrm{s})$ leading to treatment discontinuation & $7(2.8)$ & $4(1.0)$ & $4(4.6)$ & $3(2.3)$ \\
\hline One or more severe $\mathrm{AE}(\mathrm{s})$ & $10(4.0)$ & $16(4.1)$ & $8(9.2)$ & $9(7.0)$ \\
\hline One or more serious $\mathrm{AE}(\mathrm{s})$ & $9(3.6)$ & $19(4.9)$ & $7(8.0)$ & $10(7.8)$ \\
\hline Deaths & 0 & 0 & $2(2.3)$ & 0 \\
\hline Confirmed hypoglycaemic AEs ${ }^{\mathrm{b}}$ & $29(11.7)$ & $35(9.0)$ & $21(24.1)$ & $24(18.8)$ \\
\hline Events consistent with urinary tract infection ${ }^{\mathrm{c}}$ & $24(9.7)$ & $31(8.0)$ & $8(9.2)$ & $15(11.7)$ \\
\hline Events consistent with genital infection $^{\mathrm{d}}$ & $2(0.8)$ & $11(2.8)$ & 0 & $4(3.1)$ \\
\hline Events consistent with volume depletion $^{\mathrm{e}}$ & 0 & $4(1.0)$ & $1(1.1)$ & $3(2.3)$ \\
\hline Decreased renal function ${ }^{\mathrm{f}}$ & 0 & $1(0.3)$ & $3(3.4)$ & $6(4.7)$ \\
\hline Bone fracture & $1(0.4)$ & $4(1.0)$ & $1(1.1)$ & 0 \\
\hline Hyperkalaemia ${ }^{\mathrm{g}}$ & $3(1.2)$ & $2(0.5)$ & $1(1.1)$ & $2(1.6)$ \\
\hline
\end{tabular}

Data are $n(\%)$ in the treated set (randomised patients who received $\geq 1$ dose of study drug)

${ }^{\mathrm{a}}$ As defined by the investigator

${ }^{\mathrm{b}}$ Plasma glucose $\leq 3.9 \mathrm{mmol} / \mathrm{l}$ and/or requiring assistance

${ }^{\mathrm{c}}$ Based on 77 preferred terms

${ }^{\mathrm{d}}$ Based on 89 preferred terms

${ }^{\mathrm{e}}$ Based on eight preferred terms

${ }^{\mathrm{f}}$ Based on the narrow standardised Medical Dictionary for Drug Regulatory Activities (MedDRA) query 'decreased renal function'

${ }^{\mathrm{g}}$ Based on one preferred terms

weight [23]. Furthermore, dapagliflozin reduced eGFR acutely, and then maintained stable renal function over the subsequent 104 weeks of treatment [23]. Dapagliflozin treatment for 12 weeks was also shown to reduce UACR (combined microalbuminuria and macroalbuminuria) in a post hoc analysis involving patients taking baseline RAAS blockade [25]. Similar to our observations, dapagliflozin was associated with changes in UACR after adjustment for potential confounding factors. Hence, existing experimental and clinical data suggest SGLT2 inhibitors as a drug class that may lead to reductions in urinary albumin excretion independent of known drug effects on $\mathrm{BP}, \mathrm{HbA}_{1 \mathrm{c}}$ or weight.

The albuminuria-lowering effect of SGLT2 inhibition may be due to several mechanisms. First, SGLT2 inhibition achieved through either pharmacological blockade or genetic knockout models reduces renal hyperfiltration, which is considered to be a surrogate marker for intraglomerular pressure in humans [11, $12,19,26]$. Such a mechanism would be expected to reduce albuminuria independent of changes in systemic BP, similar to the effects of RAAS blockade, but through afferent vasoconstrictive effects rather than efferent vasodilatory effects. As the effects of SGLT2 inhibition on UACR occur in conjunction with small declines in eGFR over the initial 3-4 weeks in patients with type 2 diabetes, it is possible that the UACR-lowering effects of these agents are due to reduced intraglomerular pressure. Consistent with this hypothesis is the preliminary observation that SGLT2 inhibition with empagliflozin reduces calculated afferent arteriolar tone and reduces calculated glomerular capillary pressure in patients with type 1 diabetes [12]. Furthermore, in the present pooled analysis, the effect of empagliflozin on UACR remained significant and of a clinically relevant magnitude $(20-40 \%$ reduction) after controlling for on-treatment changes in $\mathrm{BP}$, weight and $\mathrm{HbA}_{1 \mathrm{c}}$. In fact, at most, only about half of the overall albuminuria-lowering effect of empagliflozin vs placebo could be explained by concomitant changes in glucose, weight or SBP (individually or together), with BP changes apparently contributing most. This supports the hypothesis that reductions in UACR were predominantly mediated via mechanisms other than those expected to result in improvements in UACR, such as reductions in glucose, BP or weight. The SGLT2 inhibitionrelated mechanisms that are independent of these metabolic changes may be explained by intrarenal haemodynamic effects characterised by alleviation of glomerular hypertension, as suggested from the reduction in renal hyperfiltration [11].

A second major mechanism that may contribute to albuminuria lowering with SGLT2 inhibition relates to the systemic vascular effects of these drugs. SGLT2 inhibition reduces BP and arterial stiffness, effects that have been associated with renal protection [27]. Our current analysis suggests that changes in SBP only modestly account for the reduction in UACR, as 
effects on UACR remained significant even after controlling for changes in this clinical variable. Our observations in patients with type 1 diabetes further support the concept that renal haemodynamic effects are disproportionately larger than BP lowering, as hyperfiltration is corrected by $\approx 20 \%$ with SGLT2 inhibition even though SBP was reduced only modestly by $3 \mathrm{mmHg}$ or $<3 \%$ [11]. Therefore, clinical observations to date suggest that direct intrarenal effects of SGLT2 inhibition contribute importantly to intraglomerular pressure, leading to decreased UACR [11].

A third relevant mechanism that may contribute to albuminuria-lowering effects of SGLT2 inhibition relates to influences on pro-inflammatory pathways, a recognised hallmark of diabetic nephropathy that can contribute to albuminuria [28]. In vitro and in vivo work has suggested that sodium glucose cotransport inhibition reduces markers of inflammation and fibrosis [18, 19, 29-31]. Although we did not measure inflammatory markers in the present trials, future studies should assess the effect of SGLT2 inhibition on pro-inflammatory and pro-fibrotic mechanisms in diabetes and associated kidney disease. Similarly, SGLT2 inhibition lowers plasma uric acid by approximately $15 \%$ [32]. In light of the putative role of uric acid as a mediator of renal and cardiovascular disease through activation of neurohormones and pro-inflammatory pathways, it is conceivable that reducing uric acid with empagliflozin may lead to salutary effects on UACR [33].

Finally, changes in effective circulating fluid volume via natriuresis are known to alter urinary albumin excretion, as demonstrated by the decline in UACR that is achieved through the use of either dietary sodium restriction or intervention with thiazide diuretics, both of which potentiate the albuminurialowering effects of RAAS inhibitors [5, 34]. As SGLT2 inhibition leads to a sustained and modest reduction in effective circulating fluid volume, the contribution of the osmotic-diuretic effect of SGLT2 inhibition to its albuminuria-lowering potential may be clinically relevant [13]. Furthermore, SGLT2 inhibition may lead to a reduction in natriuretic hormones such as atrial natriuretic peptide, which is elevated in the plasma of diabetic animals [35] and may play a role in hyperfiltration related to experimental diabetes [36].

It is important to highlight that while SGLT2 inhibition exerts renal protective effects in animals, including preservation of renal function, decreased glomerulosclerosis and tubulointerstitial fibrosis, and decreased albuminuria, these effects could be enhanced when combined with traditional ACE inhibition [37]. Whether this additive effect is achieved via haemodynamic or non-haemodynamic (i.e. anti-mitogenic or anti-inflammatory) effects is not known. However, it is tempting to speculate that similar potential benefits are possible in humans, and studies examining combination SGLT2 inhibition and RAAS inhibition in type 1 and type 2 diabetes are warranted because of the potential value of this strategy for the prevention of both primary and secondary renal complications.
Our work has limitations that need to be considered. First, these trials were designed to investigate the glucose-lowering effects of empagliflozin in patients with type 2 diabetes and, therefore, this pooled analysis of UACR should be interpreted as hypothesis generating. Similarly, the majority of patients in this relatively healthy cohort had microalbuminuria rather than macroalbuminuria. Second, urinary albumin levels were captured from predefined spot urine samples, collected as part of the continuous and comprehensive safety assessments during all five individual trials. However, we consider it a strength that safety assessments were standardised across trials, as described for other classes of glucose-lowering drugs [38], and that laboratory measurements of albuminuria and kidney function were conducted by a central laboratory. UACR is subject to significant variability, which we attempted to mitigate by including a large sample size. We were able to detect a significant effect of SGLT2 inhibition on UACR, suggesting a genuine and robust effect. Moreover, randomisation and blinding in phase III clinical trials make it unlikely that variability played an important role in either the placebo or active treatment groups. Although our sample size was adequate to assess significant effects on albuminuria across ranges of UACR, the total number of individuals with macroalbuminuria was modest. Finally, though we observed a substantial effect of empagliflozin on albuminuria reduction over 24 weeks, the effect on longer-term reduction and on glomerular function rate requires further study. However, the effect of SGLT2 inhibition on UACR with empagliflozin, dapagliflozin and canagliflozin is present by approximately 4 weeks and tends not to dissipate over time [15, 23, 24], highlighting that the uniform use of UACR endpoints at 24 weeks is acceptable for this analysis.

In conclusion, empagliflozin reduced albuminuria by a clinically meaningful amount in patients with either microor macroalbuminuria. Interestingly, changes in this renal biomarker with empagliflozin were only modestly influenced by the previously established class effects of SGLT2 inhibitors, such as reduction in $\mathrm{HbA}_{1 \mathrm{c}}$, weight and $\mathrm{BP}$, effects otherwise expected to profoundly reduce UACR. Other mechanisms to reduce albuminuria, such as non-systemic improvements in intraglomerular hypertension, may therefore be present during treatment with empagliflozin.

Acknowledgements The authors thank S. Crowe for providing support with the statistical analyses. Medical writing assistance, supported financially by Boehringer Ingelheim, was provided by E. Ng of FleishmanHillard Group during the preparation of this manuscript. The authors were fully responsible for all content and editorial decisions, were involved at all stages of manuscript development and have approved the final version.

Funding The trials that provided data for this pooled analysis were funded by the Boehringer Ingelheim \& Eli Lilly and Company Diabetes Alliance. DC was also supported by a Kidney Foundation of Canada Scholarship and a Canadian Diabetes Association-KRESCENT Program Joint New Investigator Award. 
Duality of interest DC has received speaker honoraria and acts as a consultant for Boehringer Ingelheim and BAP received operational funding with DC from Boehringer Ingelheim. P-HG has received speaker honoraria from AstraZeneca, Boehringer Ingelheim, Eli Lilly, Genzyme, Merck Sharp and Dohme, Novartis and Novo Nordisk, research grants from Eli Lilly and Roche, is an advisory board member for Abbott, AbbVie, Boehringer Ingelheim, Cebix, Eli Lilly Janssen, Medscape and Novartis and is a board member for Medix Laboratories. MvE, SSL, SK, EP and HJW are employees of Boehringer Ingelheim. SSL owns shares in Novo Nordisk A/S and shares in dynamically traded investment funds which may own stocks from pharmaceutical companies. MEC has received speaker honoraria and is a member of advisory boards for Boehringer Ingelheim, Eli Lilly and AstraZeneca.

Contribution statement DC contributed to the analysis planning and interpretation of data and drafted the manuscript. SSL, EP and MvE contributed to the analysis planning and interpretation of data, and reviewed and edited the manuscript. BAP, P-HG, MC, SK and HJW contributed to the interpretation of data, and reviewed and edited the manuscript. All authors were fully responsible for all content and editorial decisions, and approved the final version. SSL is the guarantor of this work.

\section{References}

1. Molitch ME, Adler AI, Flyvbjerg A et al (2015) Diabetic kidney disease: a clinical update from Kidney Disease: Improving Global Outcomes. Kidney Int 87:20-30

2. Rein P, Vonbank A, Saely CH et al (2011) Relation of albuminuria to angiographically determined coronary arterial narrowing in patients with and without type 2 diabetes mellitus and stable or suspected coronary artery disease. Am J Cardiol 107:1144-1148

3. de Boer IH, Afkarian M, Rue TC et al (2014) Renal outcomes in patients with type 1 diabetes and macroalbuminuria. J Am Soc Nephrol 25:2342-2350

4. Cherney DZ, Scholey JW, Miller JA (2008) Insights into the regulation of renal hemodynamic function in diabetic mellitus. Curr Diabetes Rev 4:280-290

5. Lambers Heerspink HJ, Holtkamp FA et al (2012) Moderation of dietary sodium potentiates the renal and cardiovascular protective effects of angiotensin receptor blockers. Kidney Int 82:330-337

6. Navarro-Diaz M, Serra A, Romero R et al (2006) Effect of drastic weight loss after bariatric surgery on renal parameters in extremely obese patients: long-term follow-up. J Am Soc Nephrol 17(Suppl 3):S213-S217

7. Roden M, Weng J, Eilbracht J et al (2013) Empagliflozin monotherapy with sitagliptin as an active comparator in patients with type 2 diabetes: a randomised, double-blind, placebo-controlled, phase 3 trial. Lancet Diabetes Endocrinol 1:208-219

8. Häring HU, Merker L, Seewaldt-Becker E et al (2013) Empagliflozin as add-on to metformin plus sulfonylurea in patients with type 2 diabetes: a 24-week, randomized, double-blind, placebo-controlled trial. Diabetes Care 36:3396-3404

9. Häring HU, Merker L, Seewaldt-Becker E et al (2014) Empagliflozin as add-on to metformin in patients with type 2 diabetes: a 24-week, randomized, double-blind, placebo-controlled trial. Diabetes Care 37:1650-1659

10. Kovacs CS, Seshiah V, Swallow R et al (2014) Empagliflozin improves glycaemic and weight control as add-on therapy to pioglitazone or pioglitazone plus metformin in patients with type 2 diabetes: a 24-week, randomized, placebo-controlled trial. Diabetes Obes Metab 16:147-158
11. Cherney DZI, Perkins BA, Soleymanlou N et al (2014) The renal hemodynamic effect of SGLT2 inhibition in patients with type 1 diabetes. Circulation 129:587-597

12. Škrtić M, Yang GK, Perkins BA et al (2014) Characterisation of glomerular haemodynamic responses to SGLT2 inhibition in patients with type 1 diabetes and renal hyperfiltration. Diabetologia 57:2599-2602

13. Lambers Heerspink HJ, de Zeeuw D, Wie L, Leslie B, List J (2013) Dapagliflozin a glucose-regulating drug with diuretic properties in subjects with type 2 diabetes. Diabetes Obes Metab 15:853-862

14. Cherney DZ, Perkins BA, Soleymanlou N et al (2014) Sodium glucose cotransport-2 inhibition and intrarenal RAS activity in people with type 1 diabetes. Kidney Int 86:1057-1058

15. Barnett AH, Mithal A, Manassie J et al (2014) Efficacy and safety of empagliflozin added to existing antidiabetes treatment in patients with type 2 diabetes and chronic kidney disease: a randomised, double-blind, placebo-controlled trial. Lancet Diabetes Endocrinol 2:369-384

16. de Zeeuw D, Agarwal R, Amdahl M et al (2010) Selective vitamin $D$ receptor activation with paricalcitol for reduction of albuminuria in patients with type 2 diabetes (VITAL study): a randomised controlled trial. Lancet 376:1543-1551

17. Groop PH, Cooper ME, Perkovic V, Emser A, Woerle HJ, von Eynatten M (2013) Linagliptin lowers albuminuria on top of recommended standard treatment in patients with type 2 diabetes and renal dysfunction. Diabetes Care 36:3460-3468

18. Vallon V, Gerasimova M, Rose MA et al (2014) SGLT2 inhibitor empagliflozin reduces renal growth and albuminuria in proportion to hyperglycemia and prevents glomerular hyperfiltration in diabetic Akita mice. Am J Physiol Renal Physiol 306:F194-F204

19. Tahara A, Kurosaki E, Yokono M et al (2014) Effects of sodiumglucose cotransporter 2 selective inhibitor ipragliflozin on hyperglycaemia, oxidative stress, inflammation and liver injury in streptozotocin-induced type 1 diabetic rats. J Pharm Pharmacol 66: 975-987

20. Malatiali S, Francis I, Barac-Nieto M (2008) Phlorizin prevents glomerular hyperfiltration but not hypertrophy in diabetic rats. Exp Diabetes Res 2008:305-403

21. Arakawa K, Ishihara T, Oku A et al (2001) Improved diabetic syndrome in $\mathrm{C} 57 \mathrm{BL} / \mathrm{KsJ}-\mathrm{db} / \mathrm{db}$ mice by oral administration of the $\mathrm{Na}(+)$-glucose cotransporter inhibitor T-1095. Br J Pharmacol 132: 578-586

22. Gembardt F, Bartaun C, Jarzebska N et al (2014) The SGLT2 inhibitor empagliflozin ameliorates early features of diabetic nephropathy in BTBR ob/ob type 2 diabetic mice with and without hypertension. Am J Physiol Renal Physiol 307:F317-F325

23. Kohan DE, Fioretto P, Tang W, List JF (2014) Long-term study of patients with type 2 diabetes and moderate renal impairment shows that dapagliflozin reduces weight and blood pressure but does not improve glycemic control. Kidney Int 85:962-971

24. Yale JF, Bakris G, Cariou B et al (2013) Efficacy and safety of canagliflozin in subjects with type 2 diabetes and chronic kidney disease. Diabetes Obes Metab 15:463-473

25. Heerspink HJL, Johnsson E, Gause-Nilsson I, Cain VA, Sjostrom CD (2016) Dapagliflozin reduces albuminuria in hypertensive diabetic patients using renin-angiotensin blockers. Diabetes Obes Metab 18:590-597

26. Thomson SC, Rieg T, Miracle C et al (2012) Acute and chronic effects of SGLT2 blockade on glomerular and tubular function in the early diabetic rat. Am J Physiol Regul Integr Comp Physiol 302: R75-R83

27. Cherney DZI, Perkins BA, Soleymanlou N et al (2014) The effect of empagliflozin on arterial stiffness and heart rate variability in subjects with uncomplicated type 1 diabetes mellitus. Cardiovasc Diabetol 13:28 
28. Shimizu M, Furuichi K, Toyama T et al (2013) Long-term outcomes of Japanese type 2 diabetic patients with biopsy-proven diabetic nephropathy. Diabetes Care 36:3655-3662

29. Osorio H, Coronel I, Arellano A et al (2012) Sodium-glucose cotransporter inhibition prevents oxidative stress in the kidney of diabetic rats. Oxid Med Cell Longev 2012:542042

30. Panchapakesan U, Pegg K, Gross S et al (2013) Effects of SGLT2 inhibition in human kidney proximal tubular cells - renoprotection in diabetic nephropathy? PLoS One 8, e54442

31. Terami N, Ogawa D, Tachibana $\mathrm{H}$ et al (2014) Long-term treatment with the sodium glucose cotransporter 2 inhibitor, dapagliflozin, ameliorates glucose homeostasis and diabetic nephropathy in $\mathrm{db} /$ db mice. PLoS One 9, e100777

32. Lytvyn Y, Škrtić M, Yang GK, Yip PM, Perkins BA, Cherney DZ (2015) Glycosuria-mediated urinary uric acid excretion in patients with uncomplicated type 1 diabetes mellitus. Am J Physiol Renal Physiol 308:F77-F83

33. Lytvyn Y, Perkins BA, Cherney DZ (2015) Uric acid as a biomarker and a therapeutic target in diabetes. Can J Diabetes 39:239-246
34. Vogt L, Waanders F, Boomsma F, de Zeeuw D, Navis G (2008) Effects of dietary sodium and hydrochlorothiazide on the antiproteinuric efficacy of losartan. J Am Soc Nephrol 19:999-1007

35. Allen TJ, Cooper ME, O'Brien RC, Bach LA, Jackson B, Jerums G (1990) Glomerular filtration rate in streptozotocin-induced diabetic rats. Role of exchangeable sodium, vasoactive hormones, and insulin therapy. Diabetes 39:1182-1190

36. Ortola FV, Ballermann BJ, Anderson S, Mendez RE, Brenner BM (1987) Elevated plasma atrial natriuretic peptide levels in diabetic rats. Potential mediator of hyperfiltration. J Clin Invest 80:670-674

37. Kojima N, Williams JM, Takahashi T, Miyata N, Roman RJ (2013) Effects of a new SGLT2 inhibitor, luseogliflozin, on diabetic nephropathy in T2DN rats. J Pharmacol Exp Ther 345:464-472

38. Cooper ME, Perkovic V, McGill JB et al (2015) Kidney disease end points in a pooled analysis of individual patient-level data from a large clinical trials program of the dipeptidyl peptidase 4 inhibitor linagliptin in type 2 diabetes. Am J Kidney Dis 66:441-449 